\title{
Optokinetic stimulation efficiency for sea sickness treatment
}

\author{
Alexis Maffert ${ }^{\circledR}$, Barbara Aupy \\ Head and Neck Surgery Department, HIA Clermont Tonnerre, Brest, France
}

\begin{abstract}
Background: Sea sickness is the type of motion sickness induced by maritime transport. Its prevention through optokinetic exercises is efficient. The object of this study is to evaluate the efficiency experienced by the patients as well as the impact on other motion sicknesses.

Materials and methods: One hundred and forty-one patients underwent optokinetic treatment methods between 2006 and 2014. The following parameters were studied and scored on a numeric scale: sea sickness, intensity of vomiting and ability to hold position and duties on board.

Results: Study parameters significantly improved by optokinetic reeducation method. Sea sickness was reduced by a factor of 2 . Study settings were also stable over years. Other motion sicknesses were also improved with this optokinetic stimulation.

Conclusions: Treating sea sickness by optokinetic stimulation reeducation gives good results particularly improving its related clinical manifestations, therefore allowing seamen to properly hold their functions on board. Its efficiency lasts in time and seems promising for the management of other motion sicknesses.
\end{abstract}

(Int Marit Health 2020; 71, 4: 249-252)

Key words: sea sickness, motion sickness, optokinetic stimulation, sensory conflict

\section{INTRODUCTION}

Motion sicknesses are the set of neurovegetative manifestations presented by an individual subjected to passive movements induced by any means of transportation. Sea sickness is the maritime induced motion sickness. Amarinage corresponds to the period of a few days necessary for the organism to adapt to the marine environment; beyond 2 days of physiological adaptation, one speaks of seasickness. A third of the population is sensitive to seasickness, another third will be sensitive in rough conditions and the last third during extreme conditions [1].

Balance comes as the result of central integration of multiple pieces of information from the vestibulum, the peripheral retina and from proprioceptive sensors. During movement, potential conflict arising from these different sources is the origin of motion sickness.

At sea, accelerations are intricate, unpredictable, related to the boat movement in the swell [2]. These moves have a mainly vertical component [3] with hyper and mostly hypo gravity phenomena, all the more disturbing as they occur at low frequency $(<0.3 \mathrm{~Hz})$.

Several medicinal solutions, such as antihistamines or anticholinergics, have been proposed with moderate efficiency but many side effects [4] sometimes making them unsuitable for professionals (alertness disorders).

An optokinetic stimulation reeducation technique is performed in our service and obtains good results [5]. It aims at creating sensory conflict in order to provoke and optimise the organism's adaptation.

Trendel et al. [5] showed the method's efficiency in 2010. This method also works better than placebo [6]. Reeducation takes place after clinical examination and vestibular explorations in order to check the good running of inner ears and exclude patients with underlying pathologies. Reeducation consists in 10 sessions of optokinetic stimulation to which proprioceptive perturbations and cervical movements are added. 
Table 1. Results before and after reeducation

\begin{tabular}{llll}
\hline & Before reeducation & After reeducation & P \\
\hline Seasickness intensity & & & $<0.0001$ \\
Total & $7.92[7.60-8.23]$ & $4.28[3.82-4.73]$ & $<0.0001$ \\
Male & $7.78[7.36-8.20]$ & $4.31[3.75-4.87]$ & $<0.0001$ \\
Female & $8.13[7.65-8.61]$ & $4.22[3.43-5.00]$ & $<0.0001$ \\
Vomiting intensity & & & $<0.0001$ \\
Total & $6.68[6.25-7.12]$ & $2.89[2.44-3.33]$ & $<0.0001$ \\
Male & $6.75[6.22-7.28]$ & $2.99[2.44-3.54]$ & $2.72[1.94-3.49]$ \\
Female & $6.57[5.80-7.34]$ & & $<0.0001$ \\
Working on board inability & & $3.81[3.32-4.29]$ & $<0.0001$ \\
Total & $7.20[6.81-7.59]$ & $3.60[3.02-4.19]$ & $<0.0001$ \\
Male & $7.03[6.53-7.53]$ & $4.14[3,28-5.01]$ &
\end{tabular}

Data are shown as mean and 95\% confidence interval

We were interested in what the patient felt in terms of seasickness, vomiting and ability to hold his function on board so as to assess the method's efficiency.

\section{MATERIALS AND METHODS}

This was a retrospective study concerning patients enrolled in the service from January 2006 to December 2014 with at least 1 year follow-up to the end of the reeducation. To be included, a patient had to have attended at least 8 sessions of reeducation, to accept to participate, to be of age, without underlying pathology that could disturb the response to the reeducation and to be consulting for seasickness and not for other motion sickness.

Patients were asked to answer a series of questions so that they could evaluate on a numeric scale the intensity of their sea sickness, their vomiting and their ability to work on board before and after reeducation.

We analysed the patients' data (age, sex, number of days at sea since reeducation and time since reeducation) as well as parameters such as seasickness intensity, vomiting intensity and inability to work on board.

Data were analysed with software $\mathrm{R}^{\circledR}$ version 3.2.4, with a risk threshold alpha $=0.05$.

\section{RESULTS}

\section{POPULATION}

One hundred and forty-one patients were included in the study according to de defined criteria. Among these patients, 60 were professionals (military or not).

Median age was 45 years old (from 22 to 73 years old). There was a majority of men (61\%) with a sex ratio at 1.56. The average number of days at sea since reeducation was 88.62 days with a $95 \%$ confidence interval $(955 \mathrm{Cl}$ ) of 69.15-107.29 and average time since reeducation was 3.65 years with $95 \% \mathrm{Cl}$ of 3.33-3.97 (from 1 to 9 years, median is 3 years). There was no difference between genders for age $(p=0.4544)$, number of days at sea $(p=0.1967)$ and time since reeducation ( $p=0.2038$ ).

\section{PARAMETERS ANALYSIS}

The perceived intensity of seasickness significantly improved from 7.92 to 4.28 with $p<0.0001$ (Table 1). This improvement was also observed according to gender. Observed differences between genders before and after reeducation were not significantly different from general population of the study ( $p=0.2787$ and $p=0.8458)$.

Vomiting intensity and working on board inability were also significantly improved ( $p<0.0001$ and $p<0.0001$ ) with a score from 6.68 to 2.89 for vomiting and from 7.2 to 3.81 for inability. For each sex the improvement was significant for these two parameters $(p<0.0001$ and $p<0.0001)$. There was no difference between sexes before and after reeducation ( $p=0.6982$ and $p=0.5609$ for vomiting and $p=0.2796$ and $p=0.2994$ for inability).

These parameters were analysed in function of elapsed time since reeducation. There was no significant difference in terms of age (Kruskal-Wallis test, $p=0.5827$ ) and number of days at sea $(p=0.3377)$. There was no significant difference between groups for sea sickness intensity before and after reeducation (Kruskal-Wallis test, $p=0.3332$ and $p=0.3046$ ). The same applies to vomiting (before, $p=0.9978$ and after, $p=0.3529$ ) and to working on board inability (before, $p=0.2187$ and after, $p=0.7147$ ).

Whatever elapsed time since reeducation, there was a significant improvement of sea sickness intensity per- 
Table 2. Significance of observed improvement function of elapsed time since reeducation

\begin{tabular}{|c|c|c|c|c|c|c|}
\hline $\begin{array}{l}\text { Elapsed time since reeducation } \\
\text { [years] }\end{array}$ & 1 & 2 & 3 & 4 & 5 & $\geq 6$ \\
\hline Number (male) & $10(8)$ & $34(21)$ & $37(23)$ & $24(16)$ & $11(5)$ & $25(13)$ \\
\hline $\begin{array}{l}\text { Difference of seasickness intensity } \\
\text { before and after reeducation }\end{array}$ & $p=0.0271$ & $p<0.0001$ & $p<0.0001$ & $p<0.0001$ & $p=0.0105$ & $p=0.0004$ \\
\hline $\begin{array}{l}\text { Difference of vomiting intensity before } \\
\text { and after reeducation }\end{array}$ & $p=0.0742$ & $p<0.0001$ & $p<0.0001$ & $p=0.0001$ & $p=0.041$ & $p<0.0001$ \\
\hline $\begin{array}{l}\text { Difference of working on board ability } \\
\text { before and after reeducation }\end{array}$ & $p=0.2276$ & $p<0.0001$ & $p<0.0001$ & $p<0.0001$ & $p=0.0237$ & $p<0.0001$ \\
\hline
\end{tabular}

Table 3. Improvement in other motion sickness

\begin{tabular}{lllll}
\hline & N (proportion) & Discomfort before & Discomfort after & P \\
\hline Car sickness & $48(94 \%)$ & $5.78[5.01-6.56]$ & $2.32[1.67-2.97]$ & $<0.0001$ \\
Air sickness & $26(51 \%)$ & $4.68[3.57-5.79]$ & $1.80[1.08-2.52]$ & $<0.0001$ \\
Train sickness & $25(49 \%)$ & $4.65[3.54-5.77]$ & $1.19[0.58-1.80]$ & $<0.0001$
\end{tabular}

Data are shown as number (\%) or mean and $95 \%$ confidence interval

ceived by patients (Table 2). Other parameters (vomiting and working ability) were also improved except for the 1-year elapsed group $(p=0.0742$ for vomiting intensity and $p=0.2276$ for working on board inability).

\section{OTHER MOTION SICKNESSES}

Fifty-one (including 27 female) individuals out of the 141 patients studied reported feeling an improvement for at least 1 type of motion sickness. Car sickness was incriminated by 48 (94\%) patients then air sickness (51\%) and train sickness (49\%) (Table 3).

In this patient group, the mean age was 44.37 years old, $95 \% \mathrm{Cl} 40.85-47.89$ with a median at 46 years old. There was no difference in age between this group and our study population ( $p=0.9095)$, nor between sexes ( $p=0.6878$ for men and $p=0.5964$ for women). These patients had fewer days at sea than the total study population with 58.71 days with $95 \% \mathrm{Cl} 37.26-80.16$ ( $p=0.042$ ) and the elapsed time since reeducation was not different from the study population with an average of 3.63 years and 95\% $\mathrm{Cl} 3.15-4.11$ ( $p=0.9305)$.

Among these patients who showed improvement in another motion sickness, the proportion of women is significantly higher $\left(\chi^{2}\right.$ test, $p=0.0207$ )

For each kind of motion sickness, the reported improvement was very significant $(p<0.0001)$. This improvement was also found for each gender. For males, the improvement was significant with $p<0.0001$ for car sickness, $p=0.0032$ for airsickness and $p=0.0043$ for train sickness. For females, the improvement was also significant with $p<0.0001$ for car sickness, $p=0.0094$ for airsickness and $p=0.0007$ for train.

\section{DISCUSSION}

The choice of quantifying motion sickness scores on a numeric scale has been justified by the subjectivity inherent to the Graybiel and Miller's scale [7] and the need for an easy to use evaluation method. Sea sickness presents itself with a variety of symptoms but vomiting dominates the situation and is the only easily apprehendable sign. Furthermore, even for leisure activities, seafarers have to play a part on board and the assessment of the ability to be effective on board often unfortunately takes a second place in medical approach when it is a main complaint.

This latest element needs to be noticed. The purpose that any practitioner should be aiming is the ability of the patient to ensure his function on board even though few symptoms of seasickness are still remaining. That is why even improving clinical is not good enough to evaluate the success of seasickness treatment and the reason we tried to appreciate the ability to be effective on board.

Visual stimulation alone produces symptoms of motion sickness [5]. This is also true in driving simulators or virtual reality [8]. Optokinetic stimulation relies on vestibulo-visual conflict and allows sensitisation of the vestibulum from visual stimulation $[5,8,9]$.

We had an older population than Trendel's (44.1 vs. 32.8 years old); which can be explained by the larger share of military people in Trendel's study [5]. In our study, $61 \%$ were men, which is similar to Trendel's $58.7 \%$.

The end of the studied period has been defined by an evolution in our seasickness treatment management. Over the most recent years, the cognitive part of the seasickness has been included and the patients were initiated to the op- 
timisation of the potential techniques [10]. Some sessions of reeducation were also replaced by virtual reality exercises in standing positions. More recently, new seasickness methods of management in our department use virtual reality in a sitting position associated to vertical movements thanks to a mobile deck since 2016.

A period of at least 1 year was required in the study conditions in order to evaluate benefits over time and rely on a sufficiently large number of days at sea to attest the meaningfulness of the results. With 88 days at sea on average, the seamen in the study have regularly been confronted to sea sickness. Because of the length of the reeducation method (10 sessions of optokinetic stimulation), priority should be given to patients whose exposure is frequent and for long periods.

One of the interests of this study was to be able to appreciate the stability of the benefits or the effectiveness of this method of sea sickness reeducation. We found an average of 3.65 years since reeducation but the analysis in subgroups according to the delay since reeducation showed a stability over time of the effectiveness of this method.

In the literature, there is a female predominance for seasickness (sex ratio 1.7/1) with a faster symptom appearance and more intense clinical manifestations [11-13]. We did not find any difference between genders in terms of intensity of manifestations for seasickness. Bos et al. [14] also contested this otherwise usually admitted difference. However, in the population showing improvement in other motion sicknesses, we also had a female predominance $(p=0.0207)$, that corresponds to that of the literature $[11,12]$.

The proportion of people reporting an improvement in other motion sickness was higher than we might have initially thought (nearly one third of the patients followed for seasickness). Carsickness is the most described and improved motion sickness. Other motion sicknesses improved whereas only seasickness was targeted. This illustrates the similarities of the triggering mechanisms and their interactions in the onset of clinical manifestations. It would be necessary to set up a dedicated prospective study protocol to evaluate this improvement.

\section{CONCLUSIONS}

Although longer (10 sessions) but with no risk and no side effects, sea sickness reeducation by optokinetic stimulation, progressively sensitised by the addition of head movement and disturbance of plantar somatosensory information, provides satisfactory results on vomiting intensity and seasickness intensity for people suffering from it.

This method provides long-term benefits over several years.

Nevertheless, the reduction or even cessation of vomiting does not eliminate the feeling of seasickness but it allows keeping a position on board and thus ensuring the operational capacity of seafarers. So the evaluation of the efficiency of seasickness treatment should take care of the ability of to perform their duties on board and not only the clinical results and symptoms reduction.

The optokinetic stimulation method seems to have a very interesting impact on motion sicknesses other than maritime conditions, which phenomenon should be studied with dedicated prospective protocols.

\section{CONFLICT OF INTEREST}

The authors declare that they have no conflict of interest.

\section{REFERENCES}

1. Sherman CR. Motion sickness: review of causes and preventive strategies. J Travel Med. 2002; 9(5): 251-256, doi: 10.2310/7060.2002.24145, indexed in Pubmed: 12962599.

2. Bles W, Bos JE, de Graaf B, et al. Motion sickness: only one provocative conflict? Brain Res Bull. 1998; 47(5): 481-487, doi: 10.1016/ s0361-9230(98)00115-4, indexed in Pubmed: 10052578.

3. Khalid H, Turan O, Bos J, et al. Application of the subjective verticalhorizontal-conflict physiological motion sickness model to the field trials of contemporary vessels. Ocean Engineering. 2011; 38(1): 22-33, doi: 10.1016/j.oceaneng.2010.09.008.

4. Bonne L, Loaec E, Aupy B, et al. Cinétose. EMC - Oto-rhino-laryngologie. $2017 ; 12(1): 1-5$.

5. Trendel D, Haus-Cheymol R, Erauso T, et al. Optokinetic stimulation rehabilitation in preventing seasickness. Eur Ann Otorhinolaryngol Head Neck Dis. 2010; 127(4): 125-129, doi: 10.1016/j. anorl.2010.07.006, indexed in Pubmed: 20860921.

6. Ressiot E, Dolz M, Bonne L, et al. Prospective study on the efficacy of optokinetic training in the treatment of seasickness. Eur Ann Otorhinolaryngol Head Neck Dis. 2013; 130(5): 263-268, doi: 10.1016/j.anorl.2012.03.009, indexed in Pubmed: 23562228.

7. Graybiel A, Wood CD, Miller EF, et al. Diagnostic criteria for grading the severity of acute motion sickness. Aerosp Med. 1968; 39(5): 453-455, indexed in Pubmed: 5648730.

8. Bos JE, Bles W. Motion sickness induced by optokinetic drums. Aviat Space Environ Med. 2004; 75(2): 172-174, indexed in Pubmed: 14960055.

9. Rine RM, Schubert MC, Balkany TJ. Visual-vestibular habituation and balance training for motion sickness. Phys Ther. 1999; 79(10): 949-957, indexed in Pubmed: 10498971.

10. Perreaut P. 'Comprendre et Pratiquer les Techniques d'Optimisation du Potentiel' 2014 Inter Editions.

11. Golding JF, Kadzere P, Gresty MA. Motion sickness susceptibility fluctuates through the menstrual cycle. Aviat Space Environ Med. 2005; 76(10): 970-973, indexed in Pubmed: 16235881.

12. Matchock RL, Levine ME, Gianaros PJ, et al. Susceptibility to nausea and motion sickness as a function of the menstrual cycle. Womens Health Issues. 2008; 18(4): 328-335, doi: 10.1016/j. whi.2008.01.006, indexed in Pubmed: 18485739.

13. Flanagan MB, May JG, Dobie TG. Sex differences in tolerance to visually-induced motion sickness. Aviat Space Environ Med. 2005; 76(7): 642-646, indexed in Pubmed: 16018346.

14. Bos JE, Damala D, Lewis C, etal. Susceptibilityto seasickness. Ergonomics. 2007; 50(6): 890-901, doi: 10.1080/00140130701245512, indexed in Pubmed: 17457748. 